\title{
Design and Implementation of the CNC Monitoring System Based on Internet of Things
}

\author{
Yilin Zheng ${ }^{1,2}$, Hu Lin², Qingxu Deng ${ }^{3}$, Xianli Su ${ }^{3}$ \\ ${ }^{1}$ (Graduate University of Chinese Academy of Sciences, Beijing, China) \\ ${ }^{2}$ (Shenyang Institute of Computing Technology, Chinese Academy of Sciences, Shenyang, China) \\ ${ }^{3}$ (School of Information Science \& Engineering, Northeastern University, Shenyang, China) \\ e-mail: zhengyl@sict.ac.cn
}

\begin{abstract}
Aiming at the shortcomings of the traditional CNC sensor network such as the difficult cable laying and longdistance communication signal attenuation, this paper designs the CNC monitoring system based on Internet of Things technology. The design reduces the power consumption of wireless sensor nodes and the packet loss rate of the sink node through hardware-software co-design. The Internet of Things protocol presented in this paper achieves the real-time communication between the CNC operating platform and the wireless sensor nodes. The experiment result shows that the CNC monitoring system based on Internet of Things technology can provide the temperature and vibration information for the CNC operating platform in time with the advantages of simple layout and reliable communication.
\end{abstract}

Keywords-Internet of Things; CNC monitoring system; wireless sensor nodes; low power consumption

\section{INTRODUCTION}

In the process of machining workpieces, the CNC system needs timely acquisition of physical quantities such as temperature and vibration to detect the working state of the machine tool. This demand has prompted the appearance of the CNC sensor network system. The various sensor nodes of the traditional sensor network communicate with the sink node through the line cable connection in most of the existing CNC systems. This connection simplifies the hardware design, but the cables wound in the machine bed could cause the decrease of the beauty and the difficulty of wiring. In the actual machining process, the vibration of the machine tool and the rotation of the mechanical axis could make the communication cable between the sensor nodes and the sink node broken. Moreover, the long distance of the wired communication also makes the digital signal attenuation. The shortcomings mentioned above reduce the reliability and practicability greatly, so there are very few CNC monitoring systems using the traditional sensor network for the measurement of physical quantities. With the development of wireless network technology, the Internet of Things which appears to solve the problems in the traditional sensor networks is widely used in the field of industrial monitoring [1]. In order to fill the blank in the field of CNC Internet of Things, a new CNC monitoring system is designed. This article introduces the composition of the software, hardware and network communication protocols of the monitoring system.

\section{SySTEM DESIGN}

The design of the CNC Internet of Things system whose monitoring object is a single machine uses a star network as the network topology. The system consists of the CNC operating platform, the sink node and various wireless sensor nodes (temperature, vibration, etc.). As a result of the battery power supply, the sensor nodes can be fixed in a key position of the machine, such as the spindle, the screw or the machine body. The sink node of the star network is responsible for the data communication between the CNC operating platform and the wireless sensor nodes. The operators can achieve the real-time monitoring of machine running state and control the sensor nodes through the foreground interface of the CNC operating platform. The sink node communicates with wireless senor nodes through $433 \mathrm{MHz}$ of ISM free band and exchanges data with CNC operating platform via RS232 UART protocol. The structure of the CNC monitoring system is shown in Fig. 1.

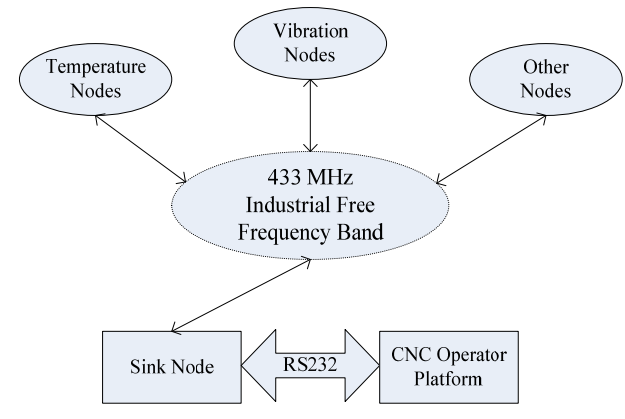

Figure 1. The structure of the CNC monitoring system

\section{HARDWARE DESIGN}

\section{A. Wireless Sensor Node Hardware Design}

Due to the limitations of the power supply and the machine bed, the sensor node hardware design should be energy-saving and compact. MSP430F2011 can meet the above requirements. In the normal mode, its power consumption is about $220 \mathrm{uA}$ and even lower than $0.5 \mathrm{uA}$ in the sleep mode. This chip uses a 14-pin plastic small-outline thin package which is only about half the size of the fingernail. In order to achieve very low-power UHF wireless transmission, the sensor node integrates a low-cost and low- 
power RF chip-CC1101. CC1101 has the size of about $4 * 4 \mathrm{~mm}$ and supports the electromagnetic wave Wake-onRadio (WOR) function for the low-power design. The sensor of the nodes uses a low-power temperature sensor DS18B20 or a low-power 3-axis vibration sensor ADXL345. The sensor nodes built by the above-mentioned components use the 3.6V lithium battery and its service lifetime is about 3 to 5 years. The remaining battery power can be real-time monitored by the power monitoring circuit. The PCB of the sensor node can be designed as small as the forefinger. The sensor node hardware design is shown in Fig. 2.

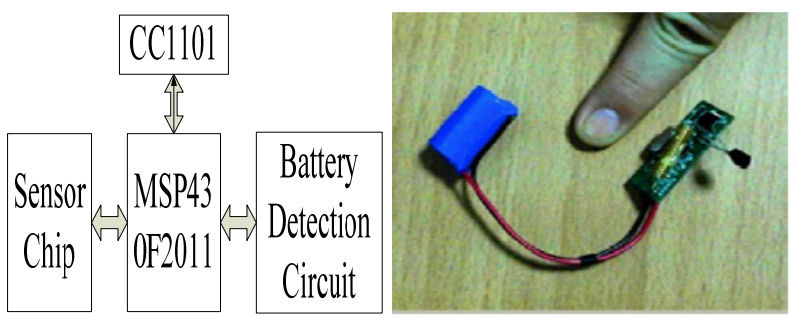

Figure 2. The sensor node hardware design

\section{B. Sink Node Hardware Design}

The sink node should forward the packets to the sensor nodes and CNC operating platform and it's in the core position of the star network [2]. For the sink node, the USB DC power is sufficient to satisfy its work demand. The microprocessor uses the microcontroller LPC1768 based on ARM Cortex-M3 core. Its main frequency is $100 \mathrm{MHz}$ and it integrates a $32 \mathrm{~K}$ SRAM and abundant peripheral components. Because of the structural characteristics of the star node, the sink node needs to send and receive a large number of packets. In order to avoid loss of receiving packets in the CC1101 sending mode, the sink node has two CC1101 chips used for packet transmission. The LPC1768 communicates with the CNC operating platform through the MAX232 chip integrated on the sink node. To take full advantages of the powerful processing capabilities and rich IO interface of LPC1768, the surplus IO pins of the sink node can be connected to other devices such as the field bus. The sink node hardware design is shown in Fig. 3.

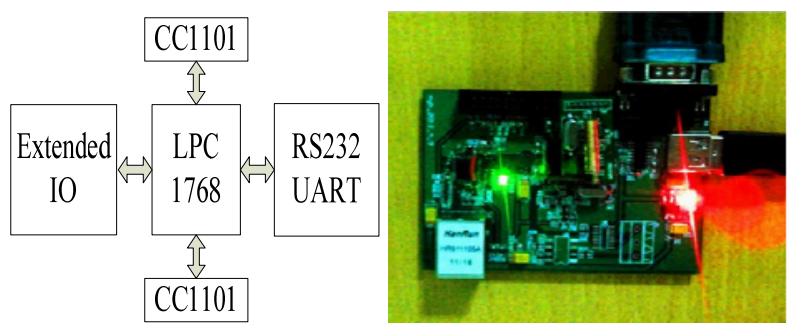

Figure 3. The sink node hardware design

\section{SOFTWARE DESIGN}

\section{A. Communication Protocol Design}

1) Communication packet design: As an important part of the monitoring system, the communication packet defines the basic structure of the communication unit between the CNC operating platform and wireless sensor nodes. The battery energy of sensor nodes is mostly consumed in the process of sending and receiving packets, so the length of packets must be as short as possible to reduce power consumption [3]. According to the need for acquisition and the direction of communication, the packets are divided into upstream packets, downstream packets and ACK packets. The upstream packet contains the original sensor information collected by the sensor nodes and its transmission direction is from sensor nodes to the CNC operating platform. Its payload is designed to be 9 bytes: the first byte is a machine number; the second byte is the sensor number; the third byte is always zero; the fourth to seventh bytes are used to store sensor data (temperature or vibration) ; the eighth byte is the battery power and the ninth byte is the accumulative total byte. The downstream packet transmitted by the CNC operating platform contains the 9-byte command information and its transmission direction is opposite to that of the upstream data packet. The third byte is the command number; the fourth byte is the command parameter; the fifth to seventh bytes are reserved to 0 ; the definition of the remaining bytes is identical to that of the upstream packet. The ACK response packet is mainly used for the confirmation of the downstream packets. Except the transmission direction, the ACK response packet has the same format and length with the downstream packet. The 9-byte communication packet format is defined as shown in Table 1.

TABLE I. THE 9-BYTE COMMUNICATION PACKET FORMAT

\begin{tabular}{|c|c|c|c|c|c|}
\hline $\begin{array}{c}\text { 1st } \\
\text { Byte }\end{array}$ & $\begin{array}{c}\text { 2nd } \\
\text { Byte }\end{array}$ & $\begin{array}{c}\text { 3rd } \\
\text { Byte }\end{array}$ & $\begin{array}{c}\text { 4th to 7th } \\
\text { Byte }\end{array}$ & $\begin{array}{c}\text { 8th } \\
\text { Byte }\end{array}$ & $\begin{array}{c}\text { 9th } \\
\text { Byte }\end{array}$ \\
\hline $\begin{array}{c}\text { Machine } \\
\text { Number }\end{array}$ & $\begin{array}{c}\text { Sensor } \\
\text { Number }\end{array}$ & $\begin{array}{c}\text { Packet } \\
\text { Type }\end{array}$ & $\begin{array}{c}\text { Data } \\
\text { (Parameters) }\end{array}$ & $\begin{array}{c}\text { Battery } \\
\text { Power }\end{array}$ & $\begin{array}{c}\text { Cumulative } \\
\text { Sum }\end{array}$ \\
\hline
\end{tabular}

2) Power saving modes: The MSP430 microprocessor chip has one normal working mode and five sleep modes. In this paper, we reduce the power consumption by switching between the normal mode and the LPM3 sleep mode. In the LPM3 sleep mode, the MSP430 power consumption can be reduced to $0.9 \mathrm{uA}$ when the microprocessor is turned off. The CC1101chip can work in one of the following four modes: sending mode, receiving mode, idle mode and sleep mode. CC1101 should spend the most time in the sleep mode to save power [4].

Using the wake-on-radio (WOR) function in the sleep mode, The CC1101 chip can be periodically wakened to monitor the potential downstream packets without the dependence on the microprocessor. To save the energy consumption of the chip, the WOR function reduces the receiving time by scanning the electromagnetic wave 
periodically. The power consumption in the sleep mode can be reduced to 900nA when using the WOR function. CC1101 will exit the sleep mode and enters the idle mode automatically when it receives correct preamble bytes in the receiving time. If the packet is not received within the receiving time, the chip will continue to sleep until the next scanning cycle. In order to make use of the WOR function, CC1101 can be set to generate an external interrupt to wake microprocessor when a synchronization word of a receiving packet is detected. The CC1101 will be switched periodically between the receiving mode and the sleep mode when the WOR function is in use, so it should be considered that CC1101 will not receive the downstream packets in the sleep mode. The schematic diagram of the WOR function is shown in Fig. 4.

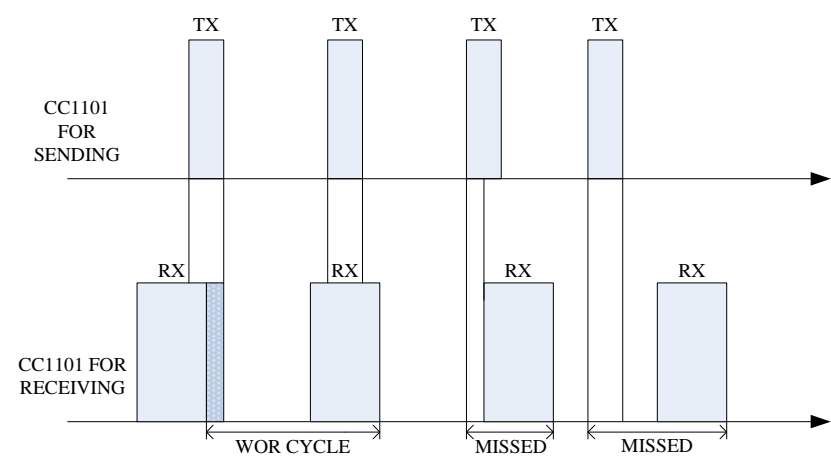

Figure 4. The schematic diagram of the WOR function

3) Detailed protocol design: The communication protocol of CNC monitoring system should be designed to ensure the real-time wireless communication and reduce the power consumption of the wireless sensor node. The sending and receiving channels of the sink nodes must be different, since the upstream packets from the wireless sensor nodes should be received only by the sink node. In this design, the receiving uses the third channel, while the sending uses the sixth channel. The two channel bandwidth is separated to avoid interference. It should be noted that CC1101 could change the operating channel only in the idle mode.

As a result of the battery-powered, the wireless sensor nodes must turn on WOR function to save power while they are waiting for receiving data. In order to solve the problem of downstream packet loss in the WOR open state, the response mechanism with ACK packets should be used when the CNC operating platform sends the downstream packet to the sensor nodes through the sink node. If the sink node receives a downstream packet in WOR receiving time, it will change the operating channel from the sixth channel to the third channel in the idle mode, and then the node enters the sending mode and sends an ACK response packet. After sending the downstream packet, the CNC operating platform begins to wait the ACK packet from the sink node. If the waiting-timeout period is expired, the downstream packet is sent again. The multiple timeout indicates the communication link failure [5]. Through the response mechanism with the ACK packets, the CNC operating platform is able to detect the quality of communication link and can ensure that each command has been received by the wireless sensor nodes.

As the sink node is supplied by the DC power, the problem of saving energy could not be considered. Taking into account the large requirement of packets exchange on the sink node, one CC1101 is in the sending mode and the other is in the receiving mode. Because the sensor data is sent periodically, the upstream packets will not change in the several periods. In order to reduce the workload of the sensor nodes as much as possible, the sink node does not need to send an ACK packet so that both CC1101 and MSP430 can stay in the sleep mode as long as possible. It is proven that this design method can extend the battery working time limit of the sensor nodes with the minimum packet loss rate [6]. The sink node enters the interrupt service routine and disables the external interrupt when it receives any kind of packet. If the execution time of interrupt service routine is particularly long, the microprocessor will refuse to respond to any external interrupt generated by many other packets so that the real-time performance of the Internet of Things system is reduced greatly. In order to make the sink node respond to the received packets in time as much as possible, LPC1768 only tests the accumulative total of the three kinds of packets mentioned above and then forwards the correct packets immediately. As a transfer station for forwarding packets, the sink node transfers the computation of the original sensor data to the more powerful CNC operating platform. This treatment reduces the run time of the LPC1768 interrupt handler and improves the transmission rate of the packets. The communication protocol of CNC monitoring system is shown in Fig. 5.

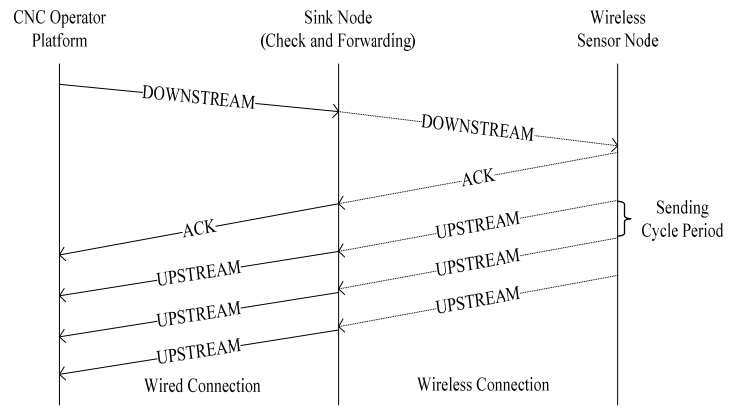

Figure 5. The communication protocol of CNC monitoring system

\section{B. Driver Design of Sensor Node}

The main goal of sensor node driver is to reduce the power consumption and communication delay of sensor nodes maximally by switching the operating mode of the microprocessor and RF chip. The MSP430 spends the most time in LPM3 sleep mode and opens the WOR function of the CC1101. If the CC1101 of the sensor node receives a 
downstream packet from the sink node in WOR's receiving time, the CC1101 sends an external interrupt signal through the GDO0 pin. This external interrupt will wake up the MSP430 from the LPM3 sleep mode so that the MSP430 turns into the normal mode to check the cumulative sum of the received packet. After analyzing the command information in the correct downstream packet, MSP430 drives the CC1101 into the sending mode. At the same time, the CC1101 sends an ACK packet to the sink node. If the sensor node needs to send the upstream packet periodically, the MSP430 should enter the watchdog interrupt handler cyclically by setting the overflow time of the watchdog timer. In the watchdog interrupt handler, the MSP430 exits from the LPM3 sleep mode and controls the CC1101 to enter the sending mode. In order to reduce the working time of MSP430 in the normal mode, the original sensor data is packaged by the upstream packet form without any other treatment. When the ACK packet or the upstream packet has been sent successfully, the CC1101 and MSP430 return to the sleep mode and wait for the next interrupt.

\section{Driver Design of Sink node}

The sink node has always been in the working state for the fast forwarding of a large number of packets. In order to make full use of the interval of the LPC1768 external interrupt, the sink node can handle other external IO requests in a while loop. For improving the packet throughput in the sink node, LPC1768 only checks the cumulative sum of received packets and hands over the subsequent processing of these packets to the next devices. If LPC1768 receives a UART interrupt, it means that the CNC operating platform should send a downstream packet to the sensor node. In the UART interrupt handler, the correct downstream packet stored in the UART receiving data buffer is sent to the sensor node by the CC1101. When LPC1768 receives an external interrupt from the GDO0 pin, it means that a sensor node should send an upstream packet to the CNC operating platform. In the external interrupt handler, the microprocessor sends the correct upstream packet to the CNC operating platform by the UART sending data buffer.

\section{Software Design of CNC Operating Platform}

For facilitating debugging and secondary development, the CNC operating platform software is divided into two parts, the middleware software for UART driver and the foreground interface. They communicate with each other through a shared database. The CNC operators can send commands to the sensor nodes through the foreground interface. The commands should be written into the history command table of the shared database, and then the middleware software sends this command with the upstream packet form through the UART. If the received packet from the UART is detected, the middleware software determines the type of the received packet based on its third byte. The middleware software deletes the corresponding command in the history command table if an ACK packet of this command is received. If an upstream packet is received, the original sensor data will be processed, and the calculated data will be stored into the collection data table of the shared database. The foreground interface only needs to display the real-time data from the shared collection data table continuously. To analyze the running state of the CNC system more accurately, the foreground interface use the waveform to display the sampled sensor data during a period of time. The foreground interface is shown in Fig. 6.

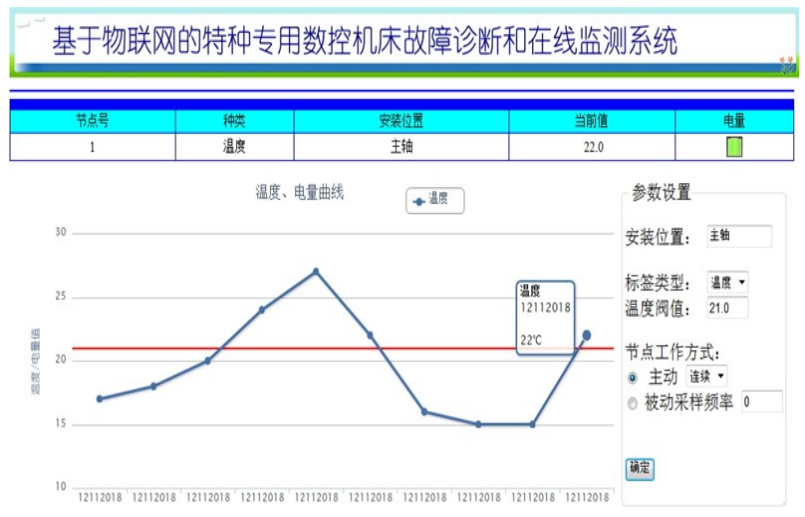

Figure 6. The foreground interface of the CNC operating platform

\section{REsults}

In this monitoring system mentioned above, the original sensor data is processed on the CNC operating platform to reduce the working time of wireless sensor nodes and the interrupt time of the sink node. Compared with the testing data processed by the MSP430 in the sensor node, it can be verified that the final data displayed on the foreground interface is correct and timely. This result shows that design presented in this paper is feasible and reliable.

\section{CONCLUSION}

The CNC monitoring system based on Internet of Things can provide fast and reliable sensor information for environmental monitoring and fault warning of CNC machine tools with the advantages of wireless sensor networks. Through the hardware-software co-design, the wireless nodes of this system can reduce power consumption in the maximal degree. It can be estimated that the battery of wireless node could work about 4 years. The design of the communication protocol improves the forwarding packet efficiency of the sink node and the real-time performance of the system. In the future work, the CNC monitoring system could be connected with the remote expert diagnosis system through the Internet connection so that the real-time monitoring and diagnosis of CNC systems can be achieved in any place connected to the Internet network [7].

\section{ACKNOWLEDGMENT}

The project is supported by the National Key Technology R\&D Program of the Ministry of Science and Technology (Shenyang Domestic Special Type CNC System Cluster Innovative Application Demonstration: No. 2012BAF13B08) and the Major National S\&T Program (High-grade CNC 
Machine Tools and Basic Manufacturing Equipment-the Innovation Platform Construction for Supporting Technology of Open Numerical Control (ONC) System: No. 2011ZX04016-071).

\section{REFERENCES}

[1] I.F. Akyildiz, W. Sankarasubramaniam, E. Cayirci, "Wireless sensor networks: a survey," Vol.38, Computer Networks, 2002, pp. 23-26.

[2] Y. Qian, "Key Technology and application of the Internet of things," Vol.8A, Telecommunications Science, 2010, pp. 118-123.

[3] K. Kredo, P. Mohapatra. "Medium access control in wireless sensor networks,” Vol.51, Computer Networks, 2007, pp. 231-242.
[4] Y.C. Tseng, Y.N. Chang, B.H. Tzeng, "Energy-efficien topolpgy cpntrol for wireless and hoc sensor networks," Vol.20, Journal of Information Science and Engineering, 2004, pp.11-16.

[5] X. Wang, S. Wang, D.W. Bi, "Dynamic sensor nodes selection strategy for wireless sensor networks," International Symposium on Communications and Information Technologies(ISCIT), 2007, pp.1135-1148.

[6] M. Kohvakka , J. Suhonen, M. Kuorilehto, V. Kaseva, M. Hannikainen, "Energy-efficient neighbor discovery protocol for mobile wireless sensor networks," Vol.7, Ad Hoc Networks, 2009, pp.25-39.

[7] S.L. Chen, Y.W. Jen, "Data fusion neural network for tool condition monitoring in CNC milling machining," Vol.40,International Journal of Machine Tools and Manufacture, 2000, pp.381-400 